\title{
Measuring the hydrostatic mass bias in galaxy clusters by combining Sunyaev-Zel'dovich and CMB lensing data
}

\author{
G. Hurier and R. E. Angulo
}

\author{
Centro de Estudios de Física del Cosmos de Aragón (CEFCA), Plaza de San Juan, 1, Planta 2, 44001 Teruel, Spain \\ e-mail: ghurier@cefca.es, rangulo@cefca.es
}

Received 26 September 2017 / Accepted 1 December 2017

\begin{abstract}
The cosmological parameters preferred by the cosmic microwave background (CMB) primary anisotropies predict many more galaxy clusters than those that have been detected via the thermal Sunyaev-Zeldovich (tSZ) effect. This discrepancy has attracted considerable attention since it might be evidence of physics beyond the simplest $\Lambda$ CDM model. However, an accurate and robust calibration of the mass-observable relation for clusters is necessary for the comparison, which has been proven difficult to obtain so far. Here, we present new constraints on the mass-pressure relation by combining tSZ and CMB lensing measurements of optically selected clusters. Consequently, our galaxy cluster sample is independent of the data employed to derive cosmological constrains. We estimate an average hydrostatic mass bias of $b=0.26 \pm 0.07$, with no significant mass or redshift evolution. This value greatly reduces the discrepancy between the predictions of $\Lambda \mathrm{CDM}$ and the observed abundance of tSZ clusters but agrees with recent estimates from tSZ clustering. On the other hand, our value for $b$ is higher than the predictions from hydrodynamical simulations. This suggests mechanisms that drive large departures from hydrostatic equilibrium and that are not included in the latest simulations, and/or unaccounted systematic errors such as biases in the cluster catalogue that are due to the optical selection.
\end{abstract}

Key words. large-scale structure of Universe - cosmic background radiation - galaxies: clusters: general galaxies: clusters: intracluster medium

\section{Introduction}

Observations of galaxy clusters via the thermal SunyaevZeldovich (tSZ) effect, the cosmic microwave background (CMB), and hydrodynamical simulations are discrepant: when we adopt the relation between total mass and gas pressure in clusters as predicted by hydrodynamic simulations, the observed abundance of $z<1$ clusters is considerably lower than what is expected for the $\Lambda$ cold dark matter (CDM) parameters preferred by CMB temperature data (Planck Collaboration XIII 2016; Planck Collaboration XX 2014).

A possible origin for the tSZ-CMB discrepancy is new physics that would modify the growth of structure between the last scattering surface and the present day. However, as shown by Salvati et al. (2017), the discrepancy cannot be solved by simple $\Lambda \mathrm{CDM}$ extensions such as massive neutrinos or a time-dependent dark energy equation of state. An alternative explanation is that the deviations from hydrostatic equilibrium are much larger than those predicted by numerical simulations.

Deviations from hydrostatic equilibrium are commonly quantified via the hydrostatic mass bias parameter, $b$, defined as the fractional difference between the true mass of a cluster and that inferred by a gas proxy assuming hydrostatic equilibrium. Given its importance for cosmology, accurately measuring $b$ and understanding the relevant astrophysics is one of the primary goals in the field of galaxy clusters.

A large number of measurements for $b$ have been performed (e.g. Medezinski et al. 2018; Sereno et al. 2017; Jimeno et al. 2017; Parroni et al. 2017; Okabe \& Smith 2016; Battaglia et al. 2016; Applegate et al. 2016; Smith et al. 2016;
Hoekstra et al. 2015; Simet et al. 2015; Israel et al. 2015; von der Linden et al. 2014; Donahue et al. 2014; Gruen et al. 2014; Mahdavi et al. 2013). These studies, mostly relying on assumptions of unbiased weak-lensing mass estimates, obtained $b \simeq 0.20 \pm 0.08$. This value is somewhat high compared to that predicted by current hydrodynamical simulations $(b \sim 0.1-0.2$, Lau et al. 2013; Hahn et al. 2015; Biffi et al. 2016), but too low to solve the CMB-tSZ discrepancy, which would require $b \geq 0.34$ (Salvati et al. 2017). It is noteworthy that a high value of $b$ was preferred by Hurier \& Lacasa (2017), who measured $b=0.30 \pm 0.07$ using a joint analysis of tSZ angular power spectrum, bispectrum, and cluster number-counts.

The above suggests that systematic errors as well as selection biases might be affecting current estimates of $b$, or conversely, that some physical processes are not captured in current hydrodynamical simulations, which would introduce further deviations from hydrostatic equilibrium in clusters. Solving this issue is crucial to derive reliable cosmological constraints and potentially detect deviations from the $\Lambda \mathrm{CDM}$ model.

The recent detection and characterisation of the gravitational lensing of CMB photons (Planck Collaboration XVII 2014), together with all-sky tSZ maps (Planck Collaboration XXII 2016), have enabled independent and robust constraints on $b$. CMB lensing has a well-determined source redshift distribution $(z \sim 1100)$, which reduces the associated systematic errors.

The correlation between tSZ and CMB lensing potential, $\phi$, over the full sky has been measured (Hill \& Spergel 2014). Unfortunately, the tSZ- $\phi$ angular power spectrum does not significantly break the degeneracy between cosmological parameters and $b$. Additionally, it has been shown that this cross-correlation 

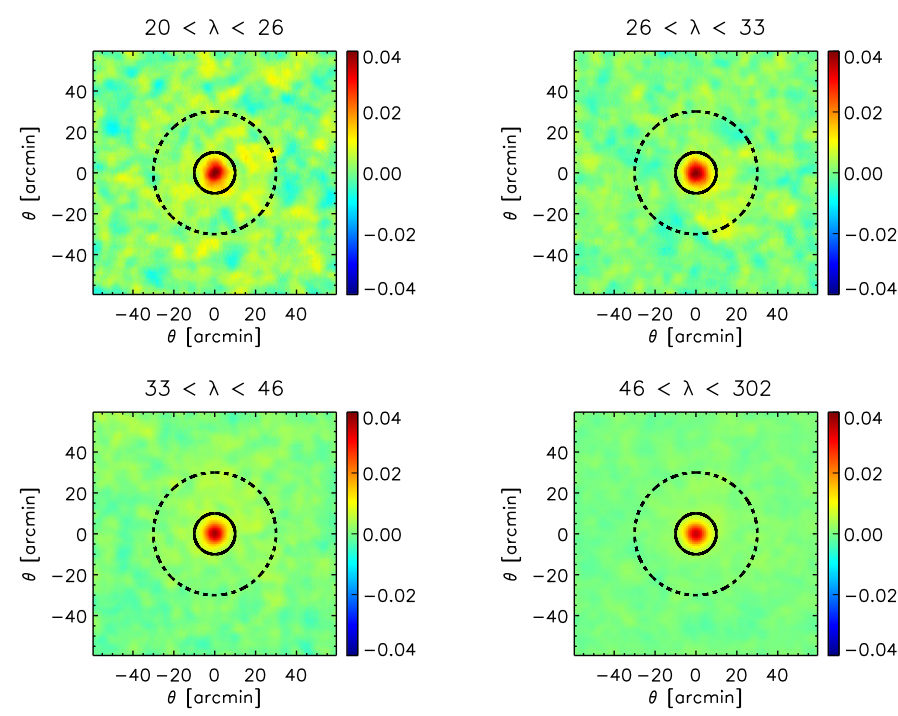

Fig. 1. Weighted stacking of the Planck tSZ MILCA map at 7' FWHM for a field of view of $2 \times 2$ degrees for four richness, $\lambda$, bins. The stacked tSZ signal is shown in arbitrary units. The solid black line delimits the region used to estimated the total tSZ flux, $Y_{\mathrm{TOT}}$, and the dashed black line delimits the region used to estimate the zero-level of the tSZ stack maps.

is significantly contaminated by cosmic infra-red background residuals (CIB, Hurier 2015). In a different approach, Melin \& Bartlett (2015) employed the CMB lensing to measure the individual masses of 61 clusters and derived $b=0.01 \pm 0.28$. Unfortunately, the low number of systems did not allow these authors to reach high statistical significance.

In the present analysis, we revisit the measurement of the hydrostatic mass bias using CMB lensing and tSZ measurements. We consider galaxy clusters identified by the algorithm called red-sequence matched-filter probabilistic percolation, or reMaPPer (Rykoff et al. 2014), on the SDSS DR8 dataset (Aihara et al. 2011). We measure the stacked tSZ signal of these clusters binned in four disjoint richness intervals. We then combine our results with the CMB-lensing measurements of Geach \& Peacock (2017), who estimated the total mass for the same clusters. With these two measurements, we place constraints on the cluster hydrostatic mass bias $b=0.26 \pm 0.07$. Finally, we explore potential redshift or mass dependencies, and we discuss the effect of our results on the tSZ-CMB discrepancy.

\section{Stacked $y$ signals of redMaPPer clusters}

The core of our analysis is to combine the recent CMB weaklensing measurements of Geach \& Peacock (2017) with suitable tSZ estimates for the gas pressure in clusters. The CMB weaklensing convergence field is given by $\kappa=\Sigma(R) / \Sigma_{\text {crit }}$, where $\Sigma(R)$ is projected mass density and $\Sigma_{\text {crit }}$ is the critical mass density,

$\Sigma_{\text {crit }}=\frac{c^{2}}{4 \pi G} \frac{D_{\mathrm{OS}}}{D_{\mathrm{OL}} D_{\mathrm{LS}}}$,

with $D_{\mathrm{OS}}, D_{\mathrm{OL}}$, and $D_{\mathrm{LS}}$ the angular diameter distance between the source and the observer, the lens and the observer, and the source and the lens, respectively. For CMB weak lensing, the source is the last scattering surface and the lens is the cluster. Geach \& Peacock (2017) used convergence maps built by the Planck satellite to estimate the average total mass, $M_{200}$, of clusters in the SDSS DR8 redMaPPer clusters catalogue
(Rykoff et al. 2014) in four richness bins. We now describe our procedure for estimating the gas pressure for the same clusters.

The intensity of the tSZ signal, $y$, is proportional to the electronic pressure, $P_{\mathrm{e}}$, of the intra-cluster medium integrated along the line of sight $l$ (Sunyaev \& Zeldovich 1972),

$y=\frac{k_{\mathrm{B}} \sigma_{\mathrm{t}}}{m_{\mathrm{e}} c^{2}} \int P_{\mathrm{e}} \mathrm{d} l$

where $k_{\mathrm{B}}$ is the Boltzmann constant, $\sigma_{\mathrm{t}}$ is the Thomson crosssection, $m_{\mathrm{e}}$ is the electron rest mass, and $c$ is the speed of light. Thus, $y$ maps can be used to estimate $P_{\mathrm{e}}$ and the hydrostatic mass bias when combined with independent cluster mass estimates.

Here, we employed the MILCA $y$ full-sky map (Hurier et al. 2013; Planck Collaboration XXII 2016) at 7' FWHM resolution (previously used in Hurier et al. 2015) in a field of view of $2 \times 2$ degrees about each cluster. It is interesting to note that $y$ maps constructed through component separation from multifrequency data, for example, MILCA, are biased tracers of the pressure since they neglect relativistic corrections (Wright 1979; Hurier 2016). This induces a significant bias on $y$ (Hurier \& Tchernin 2017), but it can be corrected for, as we describe below.

For clusters with significant contamination from radio sources, we re-computed the MILCA map and added spectral constraints to reduce the contribution from radio sources (see Hurier et al. 2013, for more details). This procedure significantly increases the noise level, therefore we applied it only to clusters with a clear contamination, as identified in the Planck $70 \mathrm{GHz}$ map.

It is well known that Planck tSZ maps suffer from infra-red emission contamination, especially from CIB (Puget et al. 1996). This contamination is particularly important for the tSZ-CMB weak-lensing cross-correlation power spectrum (Hurier 2015). However, most of this emission originates at high redshift, and by considering the CIB-leakage transfer function in the tSZ MILCA map (Planck Collaboration XXIII 2016), this contamination can be neglected for the stack of our clusters (which are at $z \leq 0.6$ ).

Following Geach \& Peacock (2017), we now stacked the tSZ signal of redMaPPer clusters split into four richness, $\lambda$, bins: [20, 26[, [26, 33[, [33, 46[, and [46, 302]. We note that the tSZ effect and the lensing have different dependencies on the cluster mass and redshift. The lensing signal scales as $M_{500} / \Sigma_{\text {crit }}$, where $M_{X}$ is the mass contained inside $R_{X}$ (the radius of a sphere with an average density equal to $X$ times the critical density of the Universe). In contrast, the tSZ flux within a $R_{500}$ aperture, $Y_{500}$, varies as $M_{500}^{1.79} E(z)^{2 / 3}$ (Planck Collaboration XX 2014).

We therefore weighted the tSZ signal associated with each cluster so that it contributed in the same way as it does to the CMB lensing. Specifically, within a given richness bin, we constructed the stacked map, $Y(\boldsymbol{\theta})$, as

$Y(\boldsymbol{\theta})=\frac{\sum_{i} w_{i} y_{i}}{\sum_{i} w_{i}}, \quad w_{i}=\frac{1}{\lambda_{i}^{0.79} E\left(z_{i}\right)^{2 / 3} \Sigma_{\text {crit }}\left(z_{i}\right)}$,

where $y_{i}$ is the tSZ MILCA map about the $i$ th cluster in the richness bin considered. We note that we assumed that $M_{500} \propto \lambda$. We also note that if the above dependences are neglected, a combined analysis would have led to significantly biased results ( $\simeq 50 \%$ on $b$ for the $[46,302]$ richness bin).

The final tSZ stacked maps are presented in Fig. 1. Overall, the tSZ signal is detected at 9 and $30 \sigma$ for the lowest and highest richness bins, respectively. 


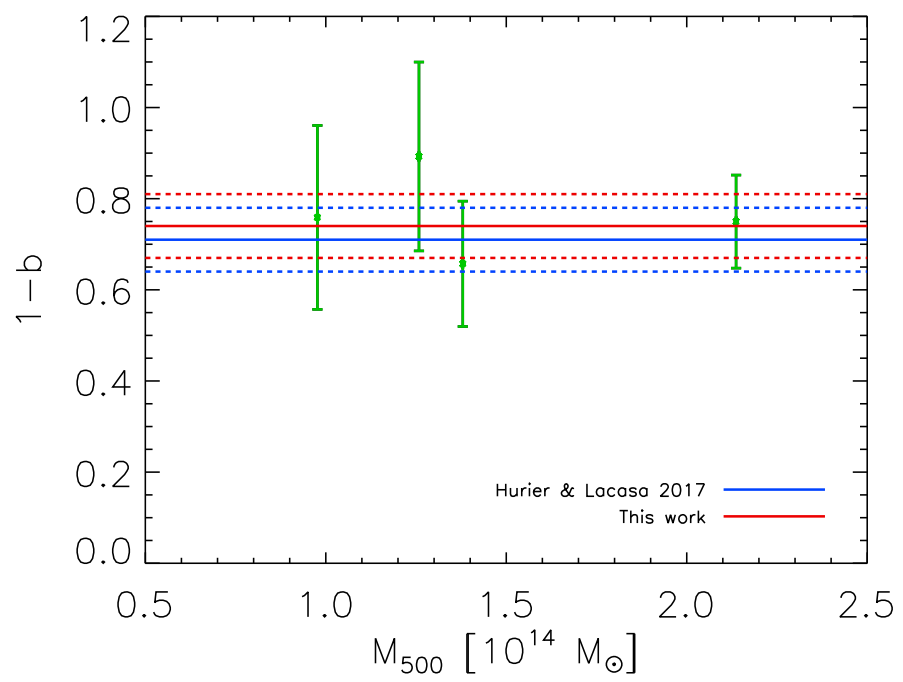

Fig. 2. Measurement of the hydrostatic mass bias derived from Planck tSZ and Planck CMB weak-lensing data (green symbols). The bestfitting average bias is shown as a red solid line, and dashed red lines shows the $1 \sigma$ uncertainty level. For comparison, we also display the constraints from Hurier \& Lacasa (2017) as blue lines.

\section{Measurement of the hydrostatic mass bias}

The second step in our analysis was to measure the average total tSZ integrated Compton parameter, $Y_{\mathrm{TOT}}$, for each richness bin. We estimated $Y_{\mathrm{TOT}}$ as the total $Y$ within an aperture of radius of $10^{\prime}$ minus the background, estimated in a $10^{\prime}$ to $30^{\prime}$ annulus. These two radii are indicated as solid and dashed black circles in Fig. 1. We note that the angular extent of clusters is expected to be smaller than $10^{\prime}$. Thus, our aperture should capture the whole signal of a cluster. For the same reason, our measurement should not be affected by any cluster off-centring.

We estimated the uncertainties in $Y_{\text {TOT }}$ using 1000 MILCA maps with different realisations of the instrumental noise (correlated inhomogeneous noise) plus CIB residuals modelled following Planck Collaboration XXIII (2016). We note that the Planck weak-lensing map is constructed using a quadratic estimator of the temperature and polarisation maps (Planck Collaboration XVII 2014). Hence, the tSZ and lensing maps should have uncorrelated noise, and we therefore neglect their effect.

We computed the hydrostatic mass bias as the ratio of the cluster mass estimated using our tSZ procedure describe above, and the CMB weak-lensing masses presented in Geach \& Peacock (2017). We converted their mass $M_{200}$ into $M_{500}$ assuming a Navarro-Frenk-White (NFW) profile (Navarro et al. 1996) and the mass-concentration relation of Duffy et al. (2008). We transformed $Y_{\text {TOT }}$ to $Y_{500}$ assuming a gNFW universal pressure profile (Arnaud et al. 2010; Planck Collaboration Int. V 2013), which gives $Y_{\mathrm{TOT}} / Y_{500}=1.79$ (Planck Collaboration XXIX 2014). Finally, we used the scaling relation $Y_{500}-M_{500}$ presented in Planck Collaboration XX (2014) to compute the cluster mass. This relation assumes hydrostatic equilibrium and has been calibrated on Planck data and X-ray observations (we note, however, that this relation does not include relativistic corrections, and in the previous section we consequently used uncorrected $y$ maps).

We present the resulting values for the hydrostatic mass bias in Fig. 2. Error bars include the uncertainty associated with both tSZ and CMB-lensing mass estimates. We derived an average bias of $b=0.26 \pm 0.07$. This value is consistent within $1 \sigma$ with previous analyses performed on CMB weak lensing (Melin \& Bartlett 2015), and gives slightly higher results than previous galaxy-galaxy weak-lensing based analyses, but the results are still consistent (see e.g. Medezinski et al. 2018; Sereno et al. 2017; Jimeno et al. 2017; Parroni et al. 2017; Okabe \& Smith 2016; Battaglia et al. 2016; Applegate et al. 2016; Smith et al. 2016; Hoekstra et al. 2015; Simet et al. 2015; Israel et al. 2015; von der Linden et al. 2014; Donahue et al. 2014; Gruen et al. 2014; Mahdavi et al. 2013. This value for $b$ is also consistent with previous results obtained from the tSZ analysis performed in Hurier \& Lacasa (2017), but it favours slightly lower values than the combined analysis of CMB and tSZ performed by Salvati et al. (2017).

We fitted for an eventual mass or redshift evolution of the hydrostatic mass bias. First we assumed the expression

$b=b_{0}+b_{1}\left(\frac{M_{500}-10^{14}\left[M_{\odot}\right]}{10^{15}\left[M_{\odot}\right]}\right)$.

We derived $b_{0}=0.26 \pm 0.13$ and $b_{1}=-0.02 \pm 1.50$, consistent with no mass evolution for the bias. Then we considered

$b=b_{2}\left(\frac{1+z}{1.38}\right)^{\alpha}$.

We derived $b_{2}=0.26 \pm 0.08$ and $\alpha=0.30 \pm 0.37$, consistent within $1 \sigma$ with no redshift evolution for the hydrostatic mass bias. Because we do not observe significant mass or redshift dependencies, we expect that our results are not significantly affected by selection effects of the redMaPPer cluster sample in the mass-redshift plane.

\section{Conclusion and discussion}

We have performed a combined analysis of tSZ and CMB weaklensing effects of SDSS DR8 redMaPPer clusters. The CMB weak lensing only depends on the integrated amount of matter along the line of sight. Thus it offers the opportunity of calibrating scaling relations related to baryonic physics with a high accuracy and little to no systematic effects.

We have inferred a value for the hydrostatic mass bias $b=$ $0.26 \pm 0.07$ with no significant redshift or mass evolution. However, the significantly different constraints derived previously from tSZ and tSZ-X-ray cross-correlation (Hurier et al. 2015; Hurier \& Lacasa 2017) may indicate that high-mass, low- $z$ clusters tend to be slightly less biased. In addition, the uncertainty in our value (mostly due to the uncertainty in CMB-lensing mass estimates) prevents us from conclusive statements about whether clusters can be modelled with a single $b$ value. Nevertheless, our value for $b$ does have implications for the tSZ-CMB discrepancy, as we show below.

In Fig. 3 we show several cosmological constraints for $\Sigma_{8}=$ $\sigma_{8}\left(\Omega_{\mathrm{m}} / 0.30\right)^{0.27}$ updated by adopting our new estimate for the hydrostatic mass bias. Specifically, we obtain $\Sigma_{8}=0.75 \pm 0.03$ for the tSZ analysis presented in Hurier \& Lacasa (2017), $\Sigma_{8}=$ $0.79 \pm 0.04$ for the tSZ-CMB weak-lensing cross-correlation (Hurier 2015), and $\Sigma_{8}=0.83 \pm 0.05$ for the X-ray-tSZ crosscorrelation (Hurier et al. 2015). For comparison, we also show the constraints derived by instead assuming $b=0.20 \pm 0.05-\mathrm{a}$ value favoured by hydrodynamical simulations.

Our constraints bracket the expected value from CMB angular power spectrum analysis, $\Sigma_{8}=0.81 \pm 0.01$, and are also consistent with the CMB weak-lensing analysis (green and blue lines in Fig. 3, respectively). All the cluster- and CMB-based constraints agree within $2 \sigma$, reducing the discrepancy between the cluster abundances and the CMB. This suggests that the 


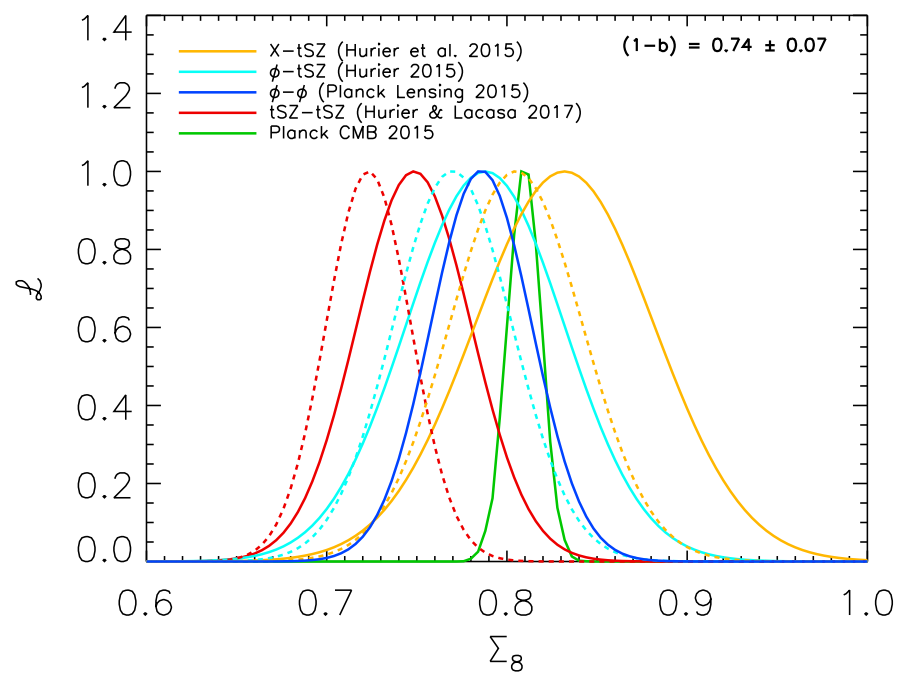

Fig. 3. Likelihood distribution of $\Sigma_{8}=\sigma_{8}\left(\Omega_{\mathrm{m}} / 0.30\right)^{0.27}$ for different analyses: tSZ angular power spectrum, bispectrum, and number count (red, Hurier \& Lacasa 2017), CMB weak lensing (dark blue, Planck Collaboration XVII 2014), CMB angular power spectrum (green, Planck Collaboration XIII 2016), tSZ-weak-lensing cross-correlation (light blue, Hurier 2015), and tSZ-X-ray cross-correlation (orange, Hurier et al. 2015). The dashed lines shows the same likelihood functions assuming $b=0.2 \pm 0.05$, whereas the solid lines uses our measured value $b=0.26 \pm 0.07$.

structure in the local and early Universe are fully consistent within the simplest $\Lambda \mathrm{CDM}$ model.

Conversely, our results now clearly disagree with cluster hydrodynamical simulations that predict low values for the hydrostatic mass bias, $b<0.2$. This would imply the lack of important physical processes in current simulations or large unaccounted-for systematic errors in observations (e.g. selection biases in the optical cluster catalogue). In the future, more sophisticated simulations with realistic mock observations as well as more accurate CMB-lensing measurements will shed light on the origin of this discrepancy.

Acknowledgements. We acknowledge the use of HEALPix (Górski et al. 2005) This project has received funding from the Spanish Ministerio de Economía and Competitividad (MINECO) through grant number AYA2015-66211-C2-2. R.E.A. acknowledges support of the European Research Council through grant number ERC-StG/716151.

\section{References}

Aihara, H., Allende Prieto, C., An, D., et al. 2011, ApJS, 193, 29 Applegate, D. E., Mantz, A., Allen, S. W., et al. 2016, MNRAS, 457, 1522 Arnaud, M., Pratt, G. W., Piffaretti, R., et al. 2010, A\&A, 517, A92 Battaglia, N., Leauthaud, A., Miyatake, H., et al. 2016, JCAP, 8, 013 Biffi, V., Borgani, S., Murante, G., et al. 2016, ApJ, 827, 112

Donahue, M., Voit, G. M., Mahdavi, A., et al. 2014, ApJ, 794, 136

Duffy, A. R., Schaye, J., Kay, S. T., \& Dalla Vecchia, C. 2008, MNRAS, 390, L64

Geach, J. E., \& Peacock, J. A. 2017, Nat. Astron., 1, 795

Górski, K. M., Hivon, E., Banday, A. J., et al. 2005, ApJ, 622, 759

Gruen, D., Seitz, S., Brimioulle, F., et al. 2014, MNRAS, 442, 1507

Hahn, O., Martizzi, D., Wu, H.-Y., et al. 2015, ArXiv e-prints [arXiv: 1509.04289]

Hill, J. C., \& Spergel, D. N. 2014, JCAP, 2, 030

Hoekstra, H., Herbonnet, R., Muzzin, A., et al. 2015, MNRAS, 449, 685

Hurier, G. 2015, A\&A, 575, L11

Hurier, G. 2016, A\&A, 596, A61

Hurier, G., \& Lacasa, F. 2017, A\&A, 604, A71

Hurier, G., \& Tchernin, C. 2017, A\&A, 604, A94

Hurier, G., Macías-Pérez, J. F., \& Hildebrandt, S. 2013, A\&A, 558, A118

Hurier, G., Douspis, M., Aghanim, N., et al. 2015, A\&A, 576, A90

Israel, H., Schellenberger, G., Nevalainen, J., Massey, R., \& Reiprich, T. H. 2015 MNRAS, 448, 814

Jimeno, P., Diego, J.-M., Broadhurst, T., De Martino, I., \& Lazkoz, R. 2017, ArXiv e-prints [arXiv: 1706.00395]

Lau, E. T., Nagai, D., \& Nelson, K. 2013, ApJ, 777, 151

Mahdavi, A., Hoekstra, H., Babul, A., et al. 2013, ApJ, 767, 116

Medezinski, E., Battaglia, N., Umetsu, K., et al. 2018, PASJ, in press [arXiv: 1706.00434$]$

Melin, J.-B., \& Bartlett, J. G. 2015, A\&A, 578, A21

Navarro, J. F., Frenk, C. S., \& White, S. D. M. 1996, ApJ, 462, 563

Okabe, N., \& Smith, G. P. 2016, MNRAS, 461, 3794

Parroni, C., Mei, S., Erben, T., et al. 2017, ApJ, 848, 114

Planck Collaboration Int. V. 2013, A\&A, 550, A131

Planck Collaboration XXIX. 2014, A\&A, 571, A29

Planck Collaboration XVII. 2014, A\&A, 571, A17

Planck Collaboration XX. 2014, A\&A, 571, A20

Planck Collaboration XIII. 2016, A\&A, 594, A13

Planck Collaboration XXIII. 2016, A\&A, 594, A23

Planck Collaboration XXII. 2016, A\&A, 594, A22

Puget, J.-L., Abergel, A., Bernard, J.-P., et al. 1996, A\&A, 308, L5

Rykoff, E. S., Rozo, E., Busha, M. T., et al. 2014, ApJ, 785, 104

Salvati, L., Douspis, M., \& Aghanim, N. 2017, ArXiv e-prints [arXiv: 1708.00697]

Sereno, M., Covone, G., Izzo, L., et al. 2017, MNRAS, 472, 1946

Simet, M., Battaglia, N., Mandelbaum, R., \& Seljak, U. 2015, in Amer. Astron. Soc. Meet. Abstr., 225, 443.04

Smith, G. P., Mazzotta, P., Okabe, N., et al. 2016, MNRAS, 456, L74

Sunyaev, R. A., \& Zeldovich, Y. B. 1972, Comm. Astrophys. Space Phys., 4, 173

von der Linden, A., Mantz, A., Allen, S. W., et al. 2014, MNRAS, 443, 1973

Wright, E. L. 1979, ApJ, 232, 348 